Clinical Research Paper

\title{
T4/N2 classification nasopharyngeal carcinoma benefit from concurrent chemotherapy in the era of intensity-modulated radiotherapy
}

\author{
Ruifei Xie ${ }^{1, *}$, Bing Xia ${ }^{1, *}$, Xuebang Zhang ${ }^{2}$, Wei Hu ${ }^{3}$, Ruping Zhao ${ }^{1}$, Congying Xie ${ }^{2}$, \\ Jianhua Wang ${ }^{3}$, Ni Zhang ${ }^{1}$ and Shixiu Wu ${ }^{1}$ \\ ${ }^{1}$ Hangzhou Cancer Hospital, Hangzhou, Zhejiang, P. R. China \\ 2 Department of Radiation Oncology, Wenzhou Medical College Cancer Center, Wenzhou, Zhejiang, P. R. China \\ ${ }^{3}$ Department of Radiation Oncology, Taizhou Central Hospital, Zhejiang, P. R. China \\ * These authors have contributed equally to this manuscript \\ Correspondence to: Shixiu Wu, email: wushixiu@medmail.com.cn
}

Keywords: nasopharyngeal carcinoma; propensity score matching; intensity modulated radiotherapy; concurrent chemotherapy Received: May 09, 2016

Accepted: September 02, 2016

Published: September 12, 2016

\section{ABSTRACT}

Although the benefits of concurrent chemotherapy (CC) in the treatment of locally advanced nasopharyngeal carcinoma (NPC) had been proven in the era of twodimensional radiotherapy, long-term efficacy and safety of using CC combined with intensity-modulated radiotherapy (IMRT) remain unclear. A retrospective analysis of 1,182 patients who underwent IMRT for clinical II-Iva NPC was performed. Propensity score matching algorithm was used to identify two matched cohorts with or without CC (264 patients per cohort). Median follow-up time was 45.6 and 43.6 months for the two cohorts. The estimated 5-year overall survival rate was $81.8 \%$ (95\% CI 76.6 87.4) in patients treated with CC and $73.7 \%(95 \% \mathrm{CI} 67.8-80.0)$ in those treated without CC, respectively (hazard ratio $0.64,95 \%$ CI $0.44-0.93 ; p=0.018$ ). The benefit of CC was mainly observed in those patients with good performance status, male, age $>48$ years, T4 and N2 classification. Grade 3/4 acute toxicities were more common in those patients administrated with CC. The grade and incidence of late salivary glands damage were also increased by $\mathrm{CC}(p=0.003)$. These findings indicated that the addition of CC significantly improved treatment outcomes of NPC patients treated with IMRT, but accompanied increased toxicities. Tailored CC and optimizing schedule of IMRT and systemic therapy were needed, provided that distant metastasis was the predominant pattern of failure in patients treated with IMRT.

\section{INTRODUCTION}

Nasopharyngeal carcinoma (NPC) is relatively uncommon worldwide, but endemic in Southeast Asia, the Arctic, and the Middle East/North Africa [1]. In 2015, it was estimated that 60,600 people would be diagnosed with NPC and 34,100 patients would die because of the disease in China, which constitutes a major public health burden [2]. Radiotherapy is the primary and only curative treatment modality in the management of NPC because of its radiosensitivity and anatomical specificity. Over the past three decades, the prognosis of NPC has dramatically improved because of advances on imaging and radiotherapy techniques, and the wide application of systemic chemotherapy $[1,3]$.

The introduction of intensity-modulated radiotherapy (IMRT) in the treatment of NPC has generated much more excitement with higher localregional control and less radiation-related sequel, due to improved coverage of tumor target and spare of normal structures. With IMRT, local-regional control rates exceeding $90 \%$ has been documented and the overall survival rate was more than $80 \%$ in recent studies [4-7]. Currently, for early stage disease (I \& IIa stage), IMRT alone achieved satisfied local control and a good survival outcome; for advanced stage disease (III \& IVa stage), 
concurrent chemotherapy (CC) combined with IMRT is often recommended empirically in clinical practice $[1,3$, 4].

However, the strategy of using concurrent chemoradiotherapy was established as the standard of care for locally advanced NPC in the two/three dimensional radiation techniques. Question remains whether IMRT combined with CC improves the therapeutic effect over and above IMRT alone. Even more concerning, incremental acute and late toxic effects were reported when CC was delivered with IMRT. The aim of this study is to investigate the role of CC in NPC patients treated with IMRT.

\section{PATIENTS AND METHODS}

\section{Patients}

We performed a retrospective analysis of 1,182 patients who underwent IMRT with or without CC for clinical II-Iva NPC from 2002 to 2009 in three cancer centers in Zhejiang Province of China (First Affiliated Hospital of Wenzhou Medical College, Hangzhou Cancer Hospital and Taizhou Central Hospital). Approval for this study was provided by the institutional review board of three hospitals, and individual patient consent was waived. All patients had complete pretreatment evaluation including clinical history, physical examination, biochemical test, fiberoptic nasopharyngoscopy with biopsy, magnetic resonance imaging (MRI) or computer tomography (CT) scan of the head and neck, chest radiography, and ultrasonography of the abdominal region. Additional investigations were performed if indicated. Stage data was retrieved from the original chart reports and was unified to the seventh edition of the International Union against Cancer/American Joint Committee on Cancer (UICC/AJCC) staging system for NPC; in case of incomplete stage information, original images were retrieved and reviewed by our study radiation oncologists.

\section{Treatment}

The techniques of planning and delivery of IMRT used in the center were similar and described previously [8]. Briefly, all patients were immobilized with specified device; contrast-enhanced planning CT scans with a 3-mm slide thickness were then obtained. All target volumes were outlined slice by slice on the axial contrast-enhanced CT images in the treatment planning system. Diagnostic MRI images were coregistered with the simulated CT images to assistant target delineation. The target volumes were defined in accordance with the International Commission on Radiation Units and Measurements Reports 50 and 62.

Simultaneous modulated accelerated radiotherapy technique was adopted. The prescribed dose was $68-70$ Gy/28-30 fractions to the gross target volume, 56-60 Gy/28-30 fractions to the high-risk clinical target volume, and 45-54 Gy/23-30 fractions to the low-risk clinical target volume. The range of radiation dose per fraction for the gross target volume was 2.3-2.5 Gy, and the high daily dose of 2.5 Gy was based on our previous study [8].

Although the benefits of $\mathrm{CC}$ in the treatment of locally advanced NPC had been proven in the era of twodimensional radiotherapy, the use of CC with IMRT was not widely accepted in this region, provided that good local-regional control with IMRT alone and high toxicity incidence rates when $\mathrm{CC}$ was delivered. With more and more evidence supported using CC for advanced NPC and accumulation of experience in the management of toxicities related to concurrent chemoradiotherapy, this strategy was accepted gradually in clinical practice but often with a reduced dose of chemotherapy. Generally, CC was delivered with cisplatin alone $\left(80 \mathrm{mg} / \mathrm{m}^{2} / \mathrm{d}\right.$ on days 1 and 22 or $25 \mathrm{mg} / \mathrm{m}^{2} / \mathrm{d}$ weekly). Adjuvant chemotherapy consisted of gemcitabine plus cisplatin, or paclitaxel/ docetaxel plus cisplatin given every three weeks for two to three cycles.

\section{Follow-up}

Follow-up evaluations occurred every 3 months during the first 2 years; every 6 months during years 3 to 5; then annually. Routine evaluations included a complete physical examination, nasopharyngoscopy or an indirect nasopharyngeal speculum examination, a biochemical profile, annual chest radiography, abdominal ultrasonography, and a head-and-neck CT/MRI. Systemic/ acute radiation adverse effects were scored by using the National Cancer Institute Common Toxicity Criteria (CTCAE, version 4.0) [9], whereas late radiation effects were evaluated according to Radiation Therapy Oncology Group/European Organisation for Research and Treatment of Cancer (RTOG/EORTC) criteria late radiation morbidity scoring schema [10].

\section{Statistical analysis}

To address the imbalance of potential confounders between groups with and without $\mathrm{CC}$, propensity score matching was used to create 2 treatment cohorts with balanced distribution of baseline characteristics [11]. The variables for the propensity score matching were choosing by first performing a logistic regression of using $\mathrm{CC}$ versus without $\mathrm{CC}$ on the following factors: age (years), sex (male vs female), ECOG performance status (0-1 vs 2), adjuvant chemotherapy (yes vs no), gross tumor volume, T-stage, N-stage, data of IMRT (2002-2005 vs 2006-2009). A revised Charlson comorbidity index, named as head and neck comorbidity index score ( $\mathrm{HN}-\mathrm{CCI}, 0$ vs $1-2$ vs 
Table 1: Baseline characteristics of nasopharyngeal carcinoma patients underwent IMRT with or without concurrent chemotherapy

\begin{tabular}{|c|c|c|c|c|c|c|c|}
\hline \multirow[b]{2}{*}{ Subgroup } & \multicolumn{4}{|c|}{ Before propensity score matching } & \multicolumn{3}{|c|}{ After propensity score matching } \\
\hline & & Without CC & With CC & $P$ value & Without CC & With CC & $P$ value \\
\hline Number & & 606 & 576 & & 264 & 264 & \\
\hline \multirow[t]{3}{*}{ Stage } & II & 192 & 50 & \multirow{3}{*}{$<0.001$} & 43 & 36 & \multirow{3}{*}{0.694} \\
\hline & III & 270 & 320 & & 145 & 149 & \\
\hline & IV & 144 & 206 & & 76 & 79 & \\
\hline \multirow[t]{4}{*}{ T stage } & 1 & 36 & 42 & \multirow{4}{*}{$<0.001$} & 12 & 12 & \multirow{4}{*}{1} \\
\hline & 2 & 267 & 122 & & 77 & 77 & \\
\hline & 3 & 182 & 247 & & 108 & 108 & \\
\hline & 4 & 121 & 165 & & 67 & 67 & \\
\hline \multirow[t]{4}{*}{ N stage } & 0 & 167 & 78 & \multirow{4}{*}{$<0.001$} & 46 & 52 & \multirow{4}{*}{0.622} \\
\hline & 1 & 225 & 195 & & 104 & 105 & \\
\hline & 2 & 172 & 257 & & 93 & 93 & \\
\hline & 3 & 42 & 46 & & 21 & 14 & \\
\hline \multirow[t]{2}{*}{ Sex } & Male & 421 & 446 & \multirow{2}{*}{0.002} & 199 & 191 & \multirow{2}{*}{0.489} \\
\hline & Female & 185 & 130 & & 65 & 73 & \\
\hline \multirow[t]{2}{*}{ ECOG PS } & $0-1$ & 280 & 281 & \multirow{2}{*}{0.407} & 121 & 139 & \multirow{2}{*}{0.139} \\
\hline & 2 & 326 & 295 & & 143 & 125 & \\
\hline \multirow{2}{*}{$\begin{array}{l}\text { Adjuvant } \\
\text { chemotherapy }\end{array}$} & No & 502 & 357 & \multirow{2}{*}{$<0.001$} & 200 & 183 & \multirow{2}{*}{0.119} \\
\hline & Yes & 104 & 219 & & 64 & 81 & \\
\hline \multirow[t]{2}{*}{$\operatorname{GTV}\left(\mathrm{cm}^{3}\right)$} & Median & 27.6 & 35.6 & \multirow{2}{*}{$<0.001$} & 31.3 & 31.9 & \multirow{2}{*}{0.8701} \\
\hline & range & $0.9-182.7$ & $1.1-250.5$ & & $1.8-150.2$ & $3.2-156.0$ & \\
\hline \multirow[t]{2}{*}{ Age (years) } & median & 50 & 47 & \multirow{2}{*}{$<0.001$} & 48 & 48 & \multirow{2}{*}{0.741} \\
\hline & range & $13-83$ & $13-75$ & & $13-74$ & $26-75$ & \\
\hline \multirow[t]{3}{*}{$\mathrm{HN}-\mathrm{CCI}$} & 0 & 290 & 257 & \multirow{3}{*}{0.176} & 121 & 120 & \multirow{3}{*}{0.596} \\
\hline & $1-2$ & 214 & 198 & & 82 & 91 & \\
\hline & 3 & 102 & 121 & & 61 & 53 & \\
\hline \multirow{2}{*}{$\begin{array}{l}\text { Date for IMRT } \\
\text { (year) }\end{array}$} & 2002-2005 & 354 & 342 & 0783 & 144 & 158 & 0.218 \\
\hline & 2006-2009 & 252 & 234 & 0.100 & 120 & 106 & \\
\hline
\end{tabular}

Abbreviation: CC, concurrent chemotherapy; ECOG PS, performance status; GTV, gross tumor volume; HN-CCI, head and neck comorbidity index score; IMRT, intensity-modulated radiotherapy.

3-6), was also included in order to control for comorbidity [12]. The potential predictors that were not statistically significant $(\mathrm{p}>0.05)$ were removed, and propensity scores were calculated from the logistic regression refit to the reduced variable group. Patients administrated with and without $\mathrm{CC}$ were then matched using a one-to-one nearest neighbour calliper of width 0.025 (maximum allowable difference in propensity scores). Only patients matched with propensity scores were included in the subsequent analyses.

Covariates balances between the two sets were examined by $t$ test (continuous variable), $\mathrm{X}^{2}$ test or Fisher's exact test (categorical variable) as appropriate. Survival rates were calculated using the Kaplan-Meier method and the log-rank test was used for comparison in the two sets.
Multivariate analyses with the Cox proportional hazards model were used to calculate hazard ratios (HR), $95 \%$ confidence intervals (CI). Two-tailed $p$-values $<0.05$ were considered statistically significant.

\section{RESULTS}

\section{Patients}

Among the 1182 patients treatment with IMRT, 576 $(48.7 \%)$ and $606(51.3 \%)$ patients were administrated with and without $\mathrm{CC}$, respectively. The significant potential factors of choosing $\mathrm{CC}$ included young age, male, 
Table 2: The estimated 5-year overall survival rates in subgroups underwent IMRT with or without concurrent chemotherapy

\begin{tabular}{lllll}
\hline Subgroup & Without CC(No $)$ & With CC(No $\left.{ }^{a}\right)$ & HR (95CI\%) & P value \\
\hline T3N0-1 & $85.3 \%(70)$ & $88.3 \%(72)$ & $0.92(0.41-2.07)$ & 0.847 \\
T4N0-1/T1-4N2 & $63.8 \%(130)$ & $77.8 \%(142)$ & $0.45(0.27-0.74)$ & 0.002 \\
T1-4N3 & $47.0 \%(21)$ & $48.2 \%(14)$ & $1.41(0.48-4.16)$ & 0.537
\end{tabular}

Abbreviations: IMRT, intensity-modulated radiotherapy; CC, concurrent chemotherapy; $\mathrm{Cl}=$ contidence interval.

The number of patients in subgroups.

Table 3: Toxic effects in nasopharyngeal carcinoma patients underwent IMRT with or without concurrent chemotherapy

\begin{tabular}{|c|c|c|c|c|c|}
\hline \multirow{3}{*}{ Subgroup } & \multicolumn{5}{|c|}{ Toxic effects, No. $(\%)$} \\
\hline & \multicolumn{2}{|c|}{ Without CC(264) } & \multicolumn{2}{|c|}{ With CC (264) } & \multirow{2}{*}{$P$ value } \\
\hline & $1-2$ & $3-4$ & $1-2$ & $3-4$ & \\
\hline Acute toxic effects during IMRT & \multicolumn{2}{|l|}{264} & \multicolumn{2}{|l|}{264} & \\
\hline Leukopenia & $150(56.8)$ & $18(6.8)$ & $136(51.5)$ & $74(28.0)$ & $<<0.001$ \\
\hline Thrombocytopenia & $79(29.9)$ & 0 & $102(38.6)$ & $16(6.1)$ & $<<0.001$ \\
\hline Mucositis & $179(67.8)$ & $74(28)$ & $154(57.5)$ & $103(39.0)$ & 0.023 \\
\hline Nausea/vomiting & $42(15.9)$ & $8(3.0)$ & $108(40.9)$ & $40(15.2)$ & $<<0.001$ \\
\hline Late toxic effects & \multicolumn{2}{|l|}{116} & \multicolumn{2}{|l|}{128} & \\
\hline Ear(deafness/otisis) & $62(53.4)$ & $6(5.2)$ & $80(62.5)$ & $8(6.3)$ & 0.258 \\
\hline Skin fibrosis & $72(62.1)$ & $3(2.6)$ & $78(60.9)$ & $3(2.3)$ & 0.994 \\
\hline Trismus & $7(6.0)$ & 0 & $9(7.0)$ & 0 & 0.801 \\
\hline Salivary glands & $48(41.2)$ & $2(1.7)$ & $78(60.9)$ & $4(3.1)$ & 0.004 \\
\hline Brain & $6(5.2)$ & $1(0.8)$ & $8(6.3)$ & $3(2.3)$ & 0.612 \\
\hline
\end{tabular}

advanced $\mathrm{T}$ and $\mathrm{N}$ stage and no adjuvant chemotherapy. After propensity score matching, $264(50 \%)$ patients treated with CC and 264 (50\%) patients treated without CC remained in the two cohorts. The included patients had highly balanced characteristics, as showed in Table 1. All subsequent analyses were based on the propensitymatched cohorts.

\section{Survival outcomes}

The median follow-up time was 45.6 months (range 3.0-132.7 months) and 43.6 months (range 3.7-138.0 months) for the patients treated with and without CC, respectively. The estimated 5-year overall survival rates were $81.8 \%(95 \%$ CI $76.6-87.4)$ in patients treated with $\mathrm{CC}$ and $73.7 \%$ (95\% CI 67.8-80.0) in those treated without $\mathrm{CC}$, respectively (HR $0 \cdot 64,95 \%$ CI $0 \cdot 44-0.93 ; p=0 \cdot 018$ ). The patients administrated with $\mathrm{CC}$ had significantly improved progression-free and locoregional failurefree survival compared with those without CC (both $p<$ $0.05)$. Although a favorable trend toward reduced distant recurrence in those patients treated with $\mathrm{CC}$, the difference did not reach a statistical significance $(p=0.094)$ (Figure $1)$.

In the subsequent subgroup analysis, over survival was chosen as the endpoint to explore the potential population who might benefit from IMRT and CC, considered that the nature and limitation of tumorcentered clinical endpoints, like tumor control or distance metastasis. The advantage of $\mathrm{CC}$ was mainly observed in those patients with good performance status, male, age $>$ 48 years, T4 and N2 classification (Figure 2). There was no clear evidence that any subgroup of patients defined by HN-CCI, date of IMRT, adjuvant chemotherapy and gross tumor volume benefited more or less from $\mathrm{CC}$ in terms of overall survival. The estimated 5-year overall survival rates for patients with T4N0-1 and T1-4N2 disease were $77.8 \%$ in the group treated with $\mathrm{CC}$ and $63.8 \%$ in those treated without CC, respectively (HR $0.50,95 \%$ CI 0.27 $0.74, p<0.002)$. There was no difference in overall survival between the two cohorts for other subgroups (T3N0-1 or T1-4N3 stage, Table 2).

\section{Pattern of first failure}

At their last follow-up visit, a total of 131 (24.8\%) patients experienced disease progression, the majority of which was distant metastasis with or without locoregional recurrences $(15.9 \%)$. In the patients treated with $\mathrm{CC}$, $17(6.4 \%)$ and 38 (14.4\%) patients had developed locoregional failures and distant metastases, respectively. In the patients treated without CC, $30(11.4 \%)$ and 46 (17.4\%) patients had developed locoregional failures and distant metastases, respectively. 

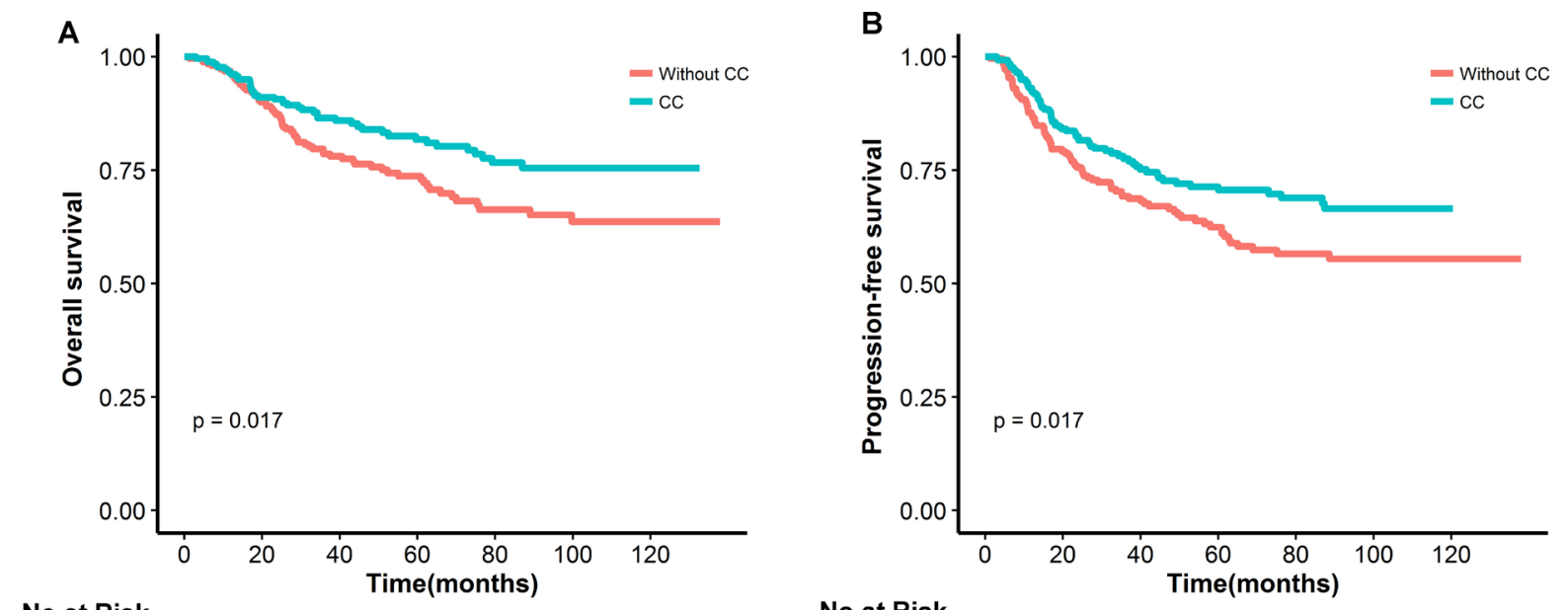

No.at Risk

No.at Risk

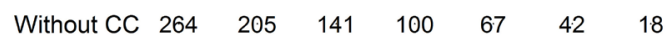

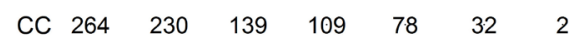

$\begin{array}{rlllllll}\text { Without CC } & 264 & 181 & 124 & 89 & 62 & 39 & 1.8 \\ \text { CC } & 264 & 213 & 126 & 97 & 71 & 29 & 1\end{array}$

C

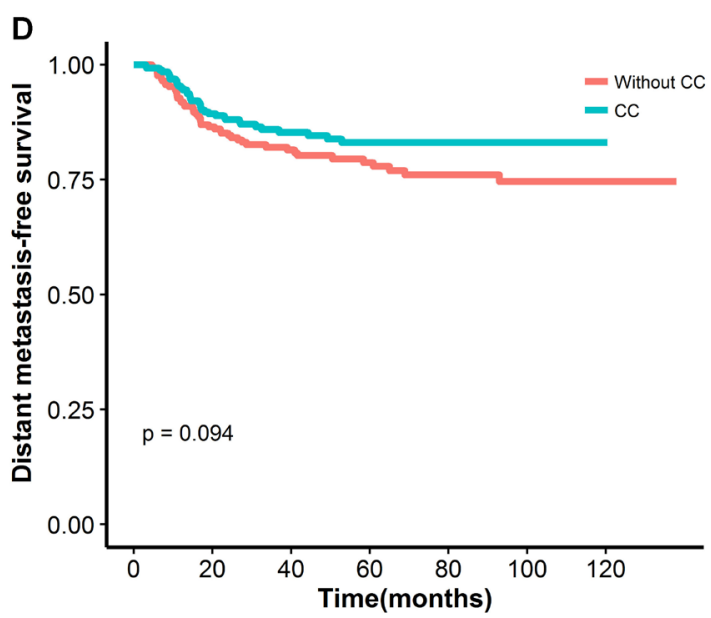

No.at Risk

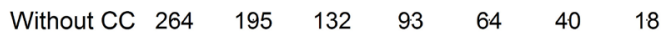

No.at Risk

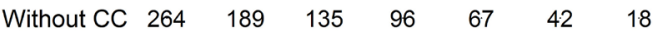

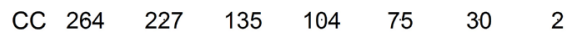

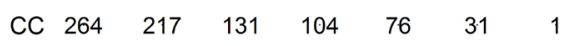

Figure 1: Comparison of overall (A), progression-free (B), locoregional recurrence-free (C) and distant metastasis-free survival curves in nasopharyngeal carcinoma patients underwent IMRT with or without concurrent chemotherapy(CC) (D).
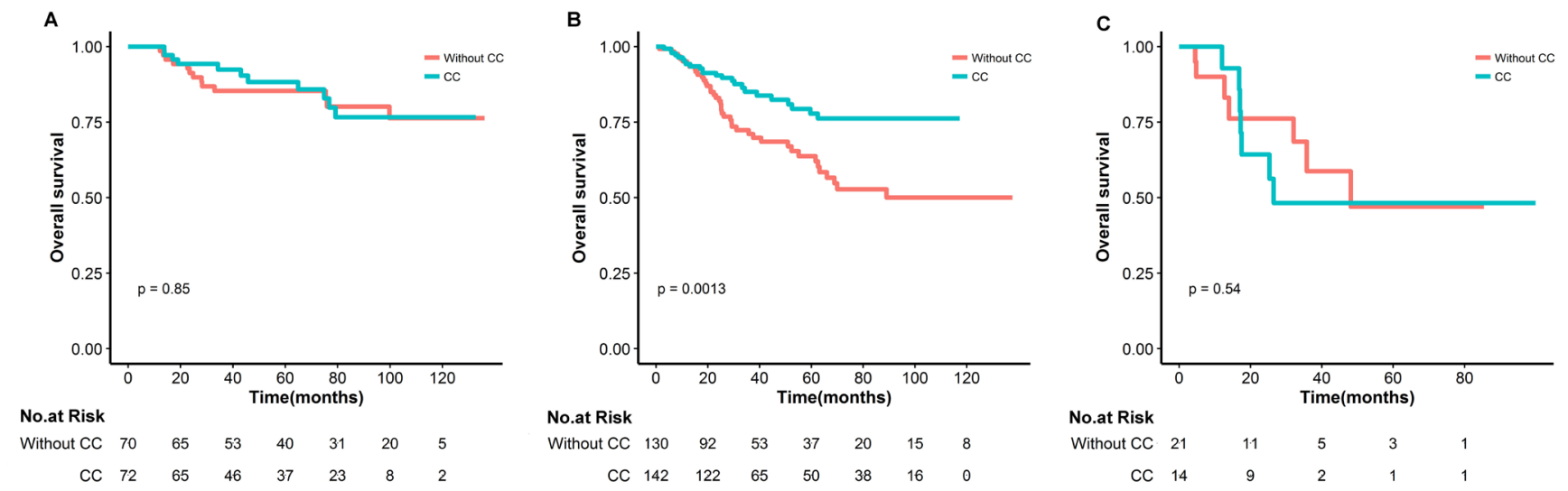

Figure 2: Kaplan-Meier survival curves in nasopharyngeal carcinoma subgroups underwent IMRT with or without concurrent chemotherapy for T3N0-1(A), T4N0-1/T1-4N2(B) and T1-4N3(D) 


\section{Toxicity}

There were no treatment-related deaths. Acute toxicities were assessed in all of the patients (Table 3). Overall, CC using cisplatin during IMRT was well tolerated; the total dose of the patients with $\mathrm{CC}$ included in this analysis ranged from $125 \mathrm{mg} / \mathrm{m}^{2}$ to $160 \mathrm{mg} / \mathrm{m}^{2}$ and no one received 1 cycle of CC. Notably, grade 3/4 of leukopenia, thrombocytopenia, mucositis and nausea/ vomiting was more common in patients delivered IMRT with $\mathrm{CC}$, compared with those without CC.

At 2 years after treatment, late toxicities were available in 128 patients with $\mathrm{CC}$ and 116 patients without. The grade and incidence of late salivary glands toxicities were increased by CC $(p=0.003)$. The worst skin fibrosis, trismus and ear toxicities were similar between the two groups who received IMRT with or without CC. There were 18 patients $(7.3 \%)$ developing MRI-diagnosed late radiation brain injury; of them, 16 patients had temporal lobe damage injury; two patients had brain stem damage.

\section{DISCUSSION}

In previous studies used conventional twodimensional radiotherapy, significant and clinically relevant improvements with the addition of $\mathrm{CC}$ had been confirmed in the treatment of NPC $[13,14]$. However, with IMRT, the long-term efficacy and safety of CC remains controversial. In a recently published MAC-NPC metaanalysis using individual patient data, the improvement of clinical outcome with CC were verified once again [15]. However, only one-fourth of the patients in the study were administrated with IMRT, and the applicability of the benefits of CC was not clear. Up to now, there is no randomized study to compare the survival outcomes and toxicities of IMRT with and without CC in patients with NPC.

In this study, the benefit of $\mathrm{CC}$ was also observed based on IMRT techniques, but the potential beneficial patients were changed to those with $\mathrm{T} 4$ or N2 classification. In the era of two-dimensional radiotherapy, the benefits of CC mainly existed in the low-risk patients

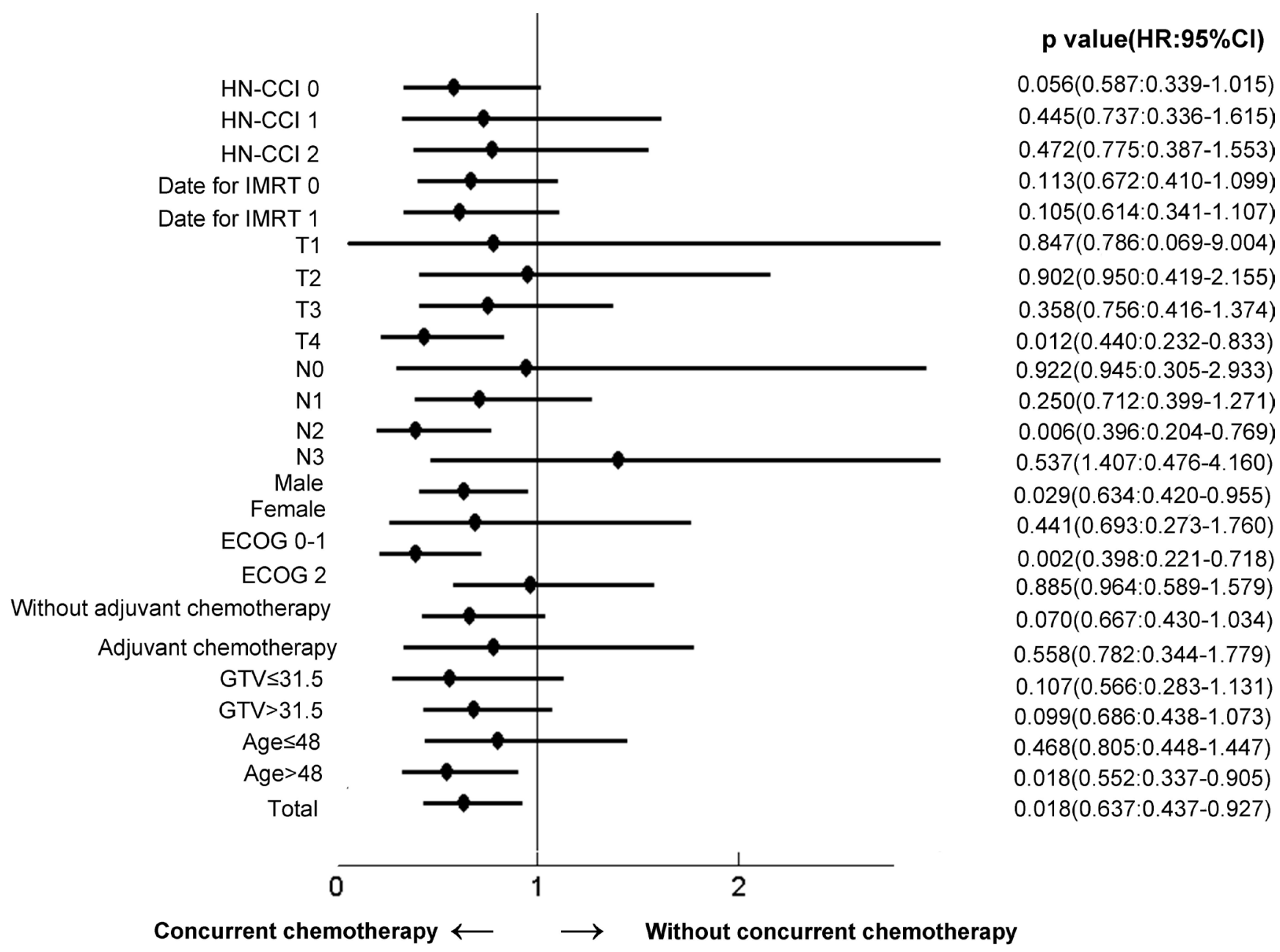

Figure 3: Effects of subgroups on overall survival in nasopharyngeal carcinoma underwent IMRT with versus without concurrent chemotherapy 
with T3-4N0-1 disease in some subgroup analysis [16-18]. The disappeared predictive value of $\mathrm{T} 3 \mathrm{~N} 0-1$ disease might be attributed to delivery of high doses to gross tumor and subclinical disease precisely, due to the advance of contemporary imaging and radiation techniques. However, T4 disease has more biologically aggressive behavior that might relate to stem cell characteristics and radiation resistance, which could not be eliminated by improved radiation dose and coverage. For those NPC patients treated with IMRT, the integration of $\mathrm{CC}$ is conducive to tumor control through a synergistic effect that different from the synchronous sensitization mechanism possibly. Compared with the findings in the era of two-dimensional radiotherapy, the emerging benefits of $\mathrm{CC}$ in the patients with N2 disease might be attributed to the improvement of radiotherapy and the application of adjuvant chemotherapy ( $73 \%$ of the patients in this study). With regard to the high-risk groups of N3 disease, the difference was not significant, indicating that $\mathrm{CC}$ was inadequate and more aggressive combined regimens might be needed.

IMRT has been shown to be superior to conventional radiotherapy in minimizing xerostomia and maintaining quality of life in many trials. However, considerable acute toxicities were accompanied when $\mathrm{CC}$ was delivered with IMRT, as observed in this study. In a recently reported study of 868 NPC patients treated with IMRT, significantly severer mucositis, xerostomia and tympanitis were experienced in those patients administrated with CC [7]. Furthermore, the high incidence rate of severe acute toxicity related to concurrent chemoradiotherapy potentially influenced subsequent adjuvant chemotherapy, leading to the difficult in complying fully to planned chemotherapy dosage. In a phase III trial aimed to evaluate the effect of adjuvant chemotherapy in the CC setting, only $63 \%$ of patients completed the planned chemotherapy, which can be used partially to explain the lack of benefits from adjuvant chemotherapy in that study [19]. Therefore, a better patient stratification for the administration of CC is clearly needed in the future studies.

Currently, distant metastasis is predominate pattern of failures in the treatment of NPC [4,5,7,20,21]. In this context, the use of systemic treatment to reduce the occurrence of metastatic relapse became more and more important, which highlight the importance of improvising patient stratification to minimize $\mathrm{CC}$ related toxicity and optimize the schedule of IMRT and systemic chemotherapy. Besides TNM staging system, a potential strategy is the incorporation of prognostic biomarkers of selecting patients at risk for locoregional relapse, which is warranted in the future studies.

Due to the nature of the study, limitations are inherent to retrospective studies, such as unknown selection bias and inconsistent interval of follow-up. It was the reason why we used propensity score matching to balance the baseline characteristics, as possible as to reduce the potential confounding factors. Also because of this, we lost the opportunity to observe the effects of CC on the compliance of adjuvant chemotherapy. In this study, we also found that patients with good performance status, male and age $>48$ years significantly benefit from $\mathrm{CC}$, which might be related to the good tolerance to aggressive therapy in those patients and some unknown biological factors in epidemic areas. However, given the nature of the study, the results should be interpreted cautiously, and need to be confirmed in prospective trials.

\section{CONCLUSIONS}

Our study found that the addition of CC significantly improved the treatment outcomes of NPC patients treated with IMRT, and the benefit was more likely to be observed in patients with T4 and N2 classification. Additionally, acute and late toxicities were increased obviously when CC was administrated with IMRT. These findings indicated that tailored $\mathrm{CC}$ and optimizing schedule of IMRT and systemic therapy were needed in future studies, provided that distant metastasis was the predominant pattern of failure in patients treated with IMRT.

\section{ACKNOWLEDGMENTS}

This work was supported by grant (2015C33268) from the public technology research and social development of Zhejiang province, by grant (2014KYA181) from the Medical and Health Research Project of Zhejiang Province, and by grant (2014A33) from the medical \& science and technology Project of Hangzhou.

\section{CONFLICTS OF INTEREST}

The authors indicated no conflicts of interest and financial disclosures.

\section{REFERENCES}

1. Chua MLK, Wee JTS, Hui EP, Chan ATC. Nasopharyngeal carcinoma. The Lancet. 2016;387:1012-1024.

2. Chen W, Zheng R, Baade PD, Zhang S, Zeng H, Bray F, Jemal A, Yu X, He J. Cancer statistics in China, 2015. CA Cancer J Clin. 2016;66:115-132.

3. Lee AWM, Ma BBY, Ng WT, Chan ATC. Management of Nasopharyngeal Carcinoma: Current Practice and Future Perspective. Journal of Clinical Oncology. 2015;33:33563364.

4. Lee N, Harris J, Garden AS, Straube W, Glisson B, Xia P, Bosch W, Morrison WH, Quivey J, Thorstad W. Intensity-Modulated Radiation Therapy With or Without Chemotherapy for Nasopharyngeal Carcinoma: Radiation Therapy Oncology Group Phase II Trial 0225. Journal of Clinical Oncology. 2009;27:3684-3690. 
5. Lin S, Lu J, Han L, Chen Q, Pan J. Sequential chemotherapy and intensity-modulated radiation therapy in the management of locoregionally advanced nasopharyngeal carcinoma: Experience of 370 consecutive cases. BMC Cancer. 2010;10:39.

6. Lee AWM, Ng WT, Chan LLK, Hung WM, Chan CCC, Sze HCK, Chan OSH, Chang ATY, Yeung RMW. Evolution of treatment for nasopharyngeal cancer - Success and setback in the intensity-modulated radiotherapy era. Radiotherapy and Oncology. 2014;110:377-384.

7. Sun X, Su S, Chen C, Han F, Zhao C, Xiao W, Deng $\mathrm{X}$, Huang S, Lin C, Lu T. Long-term outcomes of intensity-modulated radiotherapy for 868 patients with nasopharyngeal carcinoma: An analysis of survival and treatment toxicities. Radiotherapy and Oncology. 2014;110:398-403.

8. Wu S, Xie C, Jin X, Zhang P. Simultaneous modulated accelerated radiation therapy in the treatment of nasopharyngeal cancer: A local center's experience. International Journal of Radiation Oncology*Biology*Physics. 2006;66:S40-S46.

9. Chen AP, Setser A, Anadkat MJ, Cotliar J, Olsen EA, Garden BC, Lacouture ME. Grading dermatologic adverse events of cancer treatments: the Common Terminology Criteria for Adverse Events Version 4.0. J Am Acad Dermatol. Nov 2012;67:1025-1039.

10. Cox JD SJ, Pajak TF. Toxicity criteria of the Radiation Therapy Oncology Group (RTOG) and the European Organization for Research and Treatment of Cancer (EORTC). Int J Radiat Oncol Biol Phys. 1995;31:13411346.

11. Austin PC. The relative ability of different propensity score methods to balance measured covariates between treated and untreated subjects in observational studies. Med Decis Making. Nov-Dec 2009;29:661-677.

12. Bøje CR, Dalton SO, Primdahl H, Kristensen CA, Andersen E, Johansen J, Andersen LJ, Overgaard J. Evaluation of comorbidity in 9388 head and neck cancer patients: A national cohort study from the DAHANCA database. Radiotherapy and Oncology. 2014;110:91-97.

13. Lee AWM, Lau WH, Tung SY, Chua DTT, Chappell R, Xu L, Siu L, Sze WM, Leung TW. Preliminary Results of a Randomized Study on Therapeutic Gain by Concurrent Chemotherapy for Regionally-Advanced Nasopharyngeal Carcinoma: NPC-9901 Trial by the Hong Kong Nasopharyngeal Cancer Study Group. Journal of Clinical Oncology. 2005;23:6966-6975.
14. Baujat B, Audry H, Bourhis J, Chan ATC, Onat, H, Chua DTT, Kwong DLW, Al-Sarraf M, Chi KHwa, Hareyama M. Chemotherapy in locally advanced nasopharyngeal carcinoma: An individual patient data meta-analysis of eight randomized trials and 1753 patients. International Journal of Radiation Oncology*Biology*Physics. 2006;64:47-56.

15. Blanchard P, Lee A, Marguet S, Leclercq J, Ng, WT, Ma J, Chan ATC, Huang PY, Benhamou E, Zhu G. Chemotherapy and radiotherapy in nasopharyngeal carcinoma: an update of the MAC-NPC meta-analysis. The Lancet Oncology. 2015;16:645-655.

16. Wu X, Huang PY, Peng PJ, Lu LX, Han F, Wu SX, Hou $X$, Zhao HY, Huang Y, Fang WF. Long-term followup of a phase III study comparing radiotherapy with or without weekly oxaliplatin for locoregionally advanced nasopharyngeal carcinoma. Annals of Oncology. 2013;24:2131-2136.

17. Chan ATC, Leung SF, Ngan RKC, Teo PML, Lau WH, Kwan WH, Hui EP, Yiu HY, Yeo W, Cheung FY. Overall Survival After Concurrent Cisplatin-Radiotherapy Compared With Radiotherapy Alone in Locoregionally Advanced Nasopharyngeal Carcinoma. JNCI Journal of the National Cancer Institute. 2005;97:536-539.

18. Lin JC, Liang WM, Jan JS, Jiang RS, Lin AC. Another way to estimate outcome of advanced nasopharyngeal carcinoma-is concurrent chemoradiotherapy adequate? Int J Radiat Oncol Biol Phys. Sep 1 2004;60:156-164.

19. Chen L, Hu CS, Chen XZ, Hu GQ, Cheng ZB, Sun Y, Li WX, Chen YY, Xie FY, Liang SB. Concurrent chemoradiotherapy plus adjuvant chemotherapy versus concurrent chemoradiotherapy alone in patients with locoregionally advanced nasopharyngeal carcinoma: a phase 3 multicentre randomised controlled trial. Lancet Oncol. Feb 2012;13:163-171.

20. Cao C-n, Luo J-w, Gao L, Yi J, Huang X, Wang K, Zhang S, Qu Y, Li S, Cai W. Clinical outcomes and patterns of failure after intensity-modulated radiotherapy for T4 nasopharyngeal carcinoma. Oral Oncology. 2013;49:175181.

21. Ng WT, Lee $\mathrm{MCH}$, Hung WM, Choi CW, Lee, $\mathrm{KC}$, Chan OSH, Lee AWM. Clinical Outcomes and Patterns of Failure After Intensity-Modulated Radiotherapy for Nasopharyngeal Carcinoma. International Journal of Radiation Oncology*Biology*Physics. 2011;79:420-428. 